Virgilio Gallai

Paola Sarchielli

Sergio Genco

Andrea Alberti

Giovanni D'Andrea

\section{Chronic daily headache: biochemical and neurotransmitter abnormalities}

V. Gallai $(\bowtie) \cdot$ P. Sarchielli • A. Alberti Interuniversity Center for the Study of Headache and Neurotransmitter Disorders, Department of Neuroscience, University of Perugia,

Policlinico Monteluce,

Via E. Dal Pozzo, I-06126 Perugia, Italy

e-mail: gallai@unipg.it

Tel.: +39-075-573871

Fax: +39-075-5783583

\section{S. Genco}

Interuniversity Center for the Study of Headache and Neurotransmitter Disorders, Neurological Clinic I

University of Bari, Bari, Italy

\section{G. D'Andrea}

Division of Neurology,

Hospital of Este, Este (PD), Italy

\begin{abstract}
Although chronic daily headache $(\mathrm{CDH})$ represents one of the most relevant complaints of patients in headache centers, the mechanisms underlying the chronicization of head pain are poorly understood. Experimental animal models of chronic pain suggest the involvement of a functional disturbance of several neuronal pathways. The disturbances include an abnormal excitability of nociceptive fibers supplying pain-sensitive structures in the brain responsible for peripheral sensitization (chronic neurogenic inflammation), an increased responsiveness of sensory neurons of the dorsal horns in the upper spinal cord and trigeminal nucleus caudalis (central sensitization), and a functional abnormality of facilitating and inhibitory supraspinal pathways. Moreover, based on the experimental chronic pain models, the concepts of hyperalgesia and allodynia, the phenomena of wind-up and kindling, and the suggestion of a long-term potentiation (LTP) inducing a "memory of pain" also in the head have
\end{abstract}

been advocated to explain chronic head pain. However, these hypotheses have been only partially substantiated by robust findings in patients affected by CDH. In the last few years, investigation in patients with biochemical disturbances and neurotransmitter abnormalities in patients with $\mathrm{CDH}$ have been undertaken. Certain common mechanisms, but also discrepancies, have been identified between the two principal CDH forms, "transformed migraine" and chronic tension-type headache. The biochemical and neurotransmitter alterations associated with analgesic and ergotamine abuse, which often is associated with $\mathrm{CDH}$ and the more recent triptan misuse, are at the moment only partially known. The most relevant results supporting the alteration of neurotransmitter pathways related to nociception in $\mathrm{CDH}$ are reviewed.

Key words Chronic headache Central sensitization - Neurotransmitter abnormalities Experimental evidence $\cdot$ Human studies

\section{Introduction}

The neurobiology of chronic daily headache $(\mathrm{CDH})$ is scarcely known and the majority of the hypotheses on its pathophysiological mechanisms have been extrapolated from experimental animal models of pain [1]. One of the first concepts derived from animal pain models is that of primary and secondary hyperalgesias consequent to an increased afferent barrage, which induce an enhanced response to nociceptive stimuli at the site of injury and in the adjacent uninjured structures, respectively $[2,3]$. This has been related to a change in the excitability of central neurons involving first the spinal and trigeminal neurons but 
also supraspinal sites particularly in maintaining secondary hyperalgesia [4]. From the perspective of chronic head pain, changes consequent to increased afferent input from sensitized nociceptors and, perhaps previously nonactive silent nociceptors, in cranial vascular and myofascial structures, may involve a remodelling of circuitry in the upper spinal cord and also trigeminal nucleus caudalis, thereby contributing to an aberrant allodynic state responsible for persisting head pain [5].

Another hypothesis which was translated to $\mathrm{CDH}$ from experimental animal models of pain is that of central sensitization, consisting in an increased spontaneous impulse discharge of trigeminal neurons and an increased sensitivity to noxius and non-noxious stimuli and in an enlargement of the receptor fields of nociceptive neurons in trigeminal nucleus caudalis [1]. This may result in an increased activation of the trigeminovascular system. This phenomenon, well known in animal research, has a long duration and may be triggered by the "wind-up" phenomenon, which consists in an increased responsiveness of the dorsal horn and trigeminal nucleus caudalis nociceptive neurons subsequent to a repeated stimulation of $\mathrm{C}$ fibers [6]. Another suggestive hypothesis to explain chronic pain was taken from the neurophysiological model of "kindling" [7]. According to this hypothesis, repeated and persisting headache crises induce a modification in the activity of cerebral pathways involved in the modulation of pain; its reiterative firing, even in the absence of adequate trigger stimuli, may be involved in maintaining chronic pain also in the head. All these observations were assumed to potentially explain $\mathrm{CDH}$, but only few biochemical and neurotransmitter findings currently support them.

\section{Neurotransmitters involved in central mechanisms of pain}

As a consequence of an increased afferent barrage, different peptides from sensory nociceptive neurons of the trigeminovascular system and sensory neurons in the dorsal horns at $\mathrm{C}_{2}$ spinal levels, i.e. substance $\mathrm{P}(\mathrm{SP})$ and calcitonin-gene related peptide (CGRP), as well as excitatory amino acids, in particular glutamate, but also other neuroactive substances, such as galanin, somatostatin and nerve growth factors, may be responsible for the altered central nociceptive processing in the head [8]. Substance P, a neurokinin which acts principally on $\mathrm{NK}_{1}$ receptors, and CGRP which acts on its specific receptors, are important mediators of spinal and trigeminal pain transmission. Other less well known neurokinins, NK-A and NK-B, which act on $\mathrm{NK}_{2}$ and $\mathrm{NK}_{3}$ receptors respectively, may also contribute to spinal pain transmission. However, few data are available in this regard for the trigeminal systems [1,9].
A variety of $\mathrm{NK}_{1}$ receptor antagonists have been synthesized but not all investigators reported significant antihyperalgesic effects of these compounds in animal models of pain. These observations suggest that other mediators, in particular glutamate, play a major role in developing and maintaining pain; this may also be true for chronic head pain [10-13]. Receptors in the central nervous system with which glutamate interacts include ionotropic, cation-selective, ligandgated receptors and metabotropic G-protein coupled receptors [14]. The ionotropic glutamate receptors are further divided into $N$-methyl-D-aspartate (NMDA) receptors and non-NMDA receptors, which include $\alpha$-amino-3 hydroxy- $S$ methyl-4-isoxazolepropionate (AMPA) and kainate receptors named for the chemical ligands that bind to these receptors. At least 7 glutamate receptor subunits have been identified, which can assemble in a variety of conformations, thereby giving unique characteristics to different receptors on the basis of the subunit assembly [1].

Several findings suggest an interaction between NMDA receptors and sensory neurons that produce and release SP and CGRP. The former have been localized in the presynaptic terminals of fine-diameter nociceptors in the spinal cord and trigeminal nucleus caudalis, where they are believed to facilitate and prolong nociceptive input by increasing the release of neurokinins [15].

The role of NMDA receptors has been established to be central to the development and maintenance of hyperalgesia and central sensitization. Conversely, the use of NMDA receptor antagonists has been reported to be effective in experimentally induced mechanical and thermal skin hyperalgesia, in models of joint and visceral inflammation, and in models of neuropathic pain [16].

Among NMDA antagonists, non-competitive NMDA receptor channel antagonists, such as ketamine and magnesium chloride, are less effective than competitive NMDA receptors antagonists, such as CPP, in preclinical animal studies [17]. All the available data suggest that non-competitive NMDA receptor antagonists are potential antihyperalgesic but not analgesic compounds. The latter property has been, instead, attributed to the competitive NMDA receptors [18].

Non-NMDA receptor antagonists are currently available and have been documented to attenuate hyperalgesia in some experimental pain models. A recent clinical study established the efficacy of a non-AMPA/kainate receptor antagonist LY293558 on hyperalgesia and exaggerated responses to applied stimuli produced by intradermal injection of capsaicin in human volunteers [19]. This evidence, together with the observation of fewer undesiderable effects, supports the development of novel drugs with non-NMDA receptor antagonistic activity. Such antihyperalgesic compounds may be useful in the treatment of chronic pain including $\mathrm{CDH}$.

It is now known that glutamate receptors subunits can assemble to form ion channels in different ways condition- 
ing different functional characteristics of these channels. This has stimulated the development of novel, highly selective drugs that specifically act on the different glutamate receptors subunits to control pain. These compounds could be useful also in $\mathrm{CDH}$.

\section{Transduction mechanisms in nociceptive cells of dorsal horns and trigeminal nucleus caudalis}

NMDA receptor activation by glutamate released by central nociceptor terminals induces calcium entry into dorsal horn neurons and neurons of trigeminal nucleus caudalis [20]. The increased intracellular calcium induces translocation (from cytosolic to membrane-bound form) and activation of protein kinase $\mathrm{C}$ (PKC) and phosphorylation of NMDA channels which relieves the $\mathrm{Mg}^{2+}$ block on the ion channel, leading to a further increase in calcium entry [21]. The increase in the intracellular calcium may also be responsible for the induction of early genes whose main products are Fos and Jun. The two early gene products, Fos and Jun, can alter cell proteins, receptors and other peptides, and they can account for long-lasting neuronal sensitization of the trigeminal neurons which may result in increased activation of the trigeminovascular system.

In animal models of head pain, in particular, there is good evidence for Fos activation in the trigeminal nucleus caudalis [22]. Fos is considered a marker for stimulation and may be a signal for the nervous system's adaptive responses to nociceptive insults.

Calcium entry into sensory neurons in the dorsal horns of the spinal cord and in the trigeminal nucleus caudalis also induces the activation of nitric oxide (NO*) synthase leading to the synthesis of $\mathrm{NO}^{\bullet}$ and the activation of soluble guanylate cyclase. This, in turn, leads to an increase in the soluble intracellular messenger cyclic guanylate monophosphate (cGMP) [23]. Both glutamate and diffusible NO can affect the nociceptor terminal, lead to a further release of glutamate and enhance the release of neurokinins from presynaptic neurons, therefore contributing to the development of hyperalgesia and maintaining central sensitization. Due to its critical role in this regard, NO may also be the target of a therapeutic approach for controlling chronic pain, including $\mathrm{CDH}$.

\section{Supraspinal modulation of pain}

Spinal and trigeminal nociceptive input is subject to descending modulatory influences from supraspinal structures [21]. The first studies in this regard demonstrated that electrical stimulation of a number of supraspinal sites, including somatosensory cortex, periventricular gray matter, hypothalamus and thalamus, resulted in a pronounced analgesia. The most effective sites involved in stimulusinduced analgesia are located in the brain stem, i.e. in the ventrolateral column of the periacqueductal gray, pontine locus coeruleus and rostral ventromedial medulla (RVM) $[24,25]$. The terminals of the descending pathways from these structures conveyed in the dorsolateral funiculi and ended in the laminae I, II and V of the trigeminal nucleus caudalis and in the laminae I-III and V-VII of the primary afferent nociceptors and cell bodies of second-order ascending nociceptive neurons.

Although descending pain modulatory systems were originally considered to inhibit trigeminal and spinal nociceptive sensory input, other descending systems from supraspinal sites have been demonstrated to be involved in facilitation of spinal nociceptive transmission and conveyed bilaterally in the ventrolateral funiculi. Additionally some structures, such as RVM, are involved both in inhibition and facilitation of spinal nociceptive transmission, depending on the intensity of the stimulus (high intensities are inhibitory, low intensities are facilitating). In the RVM there are "off cells" which inhibit and "on cells" that facilitate nociception. The descending pathways from these cells which mediate these opposite influences are anatomically, physiologically and pharmacologically distinct [26]. They modulate the activity of the trigeminal and dorsal horn neurons. In particular, increased activity of modulatory fibers originating from on cells may enhance the response to both painful and non-painful stimuli and contribute to control central sensitization.

The spinal mediators of descending nociceptive inhibitory influences include opioids, serotonin (5-HT), norepinephine (NE) and acetylcholine (ACh). Descending facilitatory influences are mediated, at least in part, by spinal serotonin (5-HT $)$, cholecystokinin, and $K$ opioid. Descending facilitating modulation involves activation of NMDA and neurotensin receptors as well as production of $\mathrm{NO}^{*}$, particularly in the RVM. Moreover, in some circumstances enhanced descending inhibitory influences are associated with persistent nociceptive input and involve nonNMDA receptors in the RVM [18].

The notion that activation of descending medullospinal projection (as well as peripheral nociceptors) modulates central sensitization and hyperalgesia is supported by numerous studies examining various animal models of pain. This concept could be translated to chronic head pain.

\section{Central sensitization in CDH}

Central sensitization, consisting in increased neural 
excitability at different levels of the central nervous system (CNS), due to a prolonged nociceptive input from vascular or pericranial myofascial tissues, is believed to play a role in $\mathrm{CDH}$, both in episodic migraine and that evolving from a previous episodic tension-type headache. Recent data suggest an alteration in central nociceptive circuits in patients with chronic headache. Fusco et al. [27] investigated the two painful sensations induced by appropriate nociceptive stimuli, the "first pain", i.e. the initial pain sensation depending on the applied stimulus and the "second pain" that is characterized by a deeper painful sensation, more difficult to localize and with variable duration. Second pain was characterized by the phenomenon of temporal summation, i.e. the increase in its intensity due to repeated painful stimuli. This phenomenon is considered to be the physiological correlate of the activation of central excitatory circuits. The entity and characteristics of second pain were investigated by the authors in patients affected by transformed migraine, patients with chronic headache ab initio and in patients with chronic tension-type headache. They showed that second pain was significantly amplified and particularly accentuated in all chronic headache patient groups, whereas temporal summation of second pain was altered in patients affected by transformed migraine and chronic headache ab initio (both groups suffered from $\mathrm{CDH}$ with superimposed migraine attacks) but not by CTTH. Ergotamine withdrawal in chronic migraine patients who abused this drug induced a decrease of second pain.

More recently, Fusco [28] demonstrated that the oral administration of dextromethorphan, a weak antagonist of NMDA receptors, reduced the increased temporal summation of second pain. This effect was further augmented by the contemporary administration of magnesium which inhibits excitatory amino acid transmission in central neuronal circuits involved in the processing of nociceptive information. On the basis of these observations, the authors suggested the use of both drugs as a potential therapeutic approach for transformed migraine. He also proposed that proparacetamol, the precursor of acetaminophen, modulates excitatory amino acid receptors in central nociceptive pathways in these patients.

Based on the assumption that chronic daily headache with analgesic drug abuse is characterized by increased activity of the excitatory amino acid pathway, Nicolodi et al. [29] stated that the administration of NMDA receptor antagonists, such as ketamine, or of inhibitors of the synthesis or the release of excitatory amino acids, such as gabapentin, enables the analgesic-drug dependence associated with chronic daily migraine to be overcome without any physical abstinence signs. Follow-up of these patients indicated that negative modulators of pathways mediated by excitatory amino acids can induce a stable clinical benefit.
Although promising from a theoretical point of view, NMDA receptor antagonists produce undesirable psychotomimetic and motor side effects which limit their widespread use for $\mathrm{CDH}$ in clinical practice.

\section{Nitric oxide, central sensitization and CDH}

Nitric oxide has been demonstrated to play a crucial role in central sensitization in animal models of pain [30]. This NO-mediated sensitization may be a common denominator in the pain mechanisms of primary headaches. Supersensitivity to glyceryl trinitrate (GTN) by a nitric oxide donor was demonstrated in migraine even in cases of frequent attacks [31], but was not investigated in patients with transformed migraine. Using the GTN model of experimental headache, the intensity, quality and time profile of headache after infusion of GTN $(0.5 \mu \mathrm{g} / \mathrm{kg}$ per minute for $20 \mathrm{~min}$ ) were recently studied by Ashina et al. [32] in 16 patients affected by CTTH in comparison with 16 healthy controls. Subjects were randomized to receive intravenous infusion of GTN or placebo on 2 headachefree days separated by at least one week [32]. In CTTH patients, the area under the curve (AUC, intensity $x$ duration) on a GTN day was significantly higher than on a placebo day $(p<0.008)$. Moreover on the day of GTN, the AUC of the patients was significantly greater than that of healthy controls. In control subjects, peak pain intensity occurred $20 \mathrm{~min}$ after the start of infusion; in CTTH patients it occurred 8 hours after GTN infusion. In the latter group, NO induced a biphasic response with an immediate headache and a delayed headache, which has the same characteristics of spontaneous attacks in patients.

Based on the findings obtained in experimental models of pain and on the demonstration that NOS inhibition reduces central sensitization in models of chronic pain, the analgesic $N(\mathrm{G})$ monomethyl L-arginine (L-NMMA), a nonselective NOS inhibitor, was demonstrated to be effective in relieving pain in CTTH patients [33]. A significant reduction in mean pain score, measured using visual and verbal analogical scales, was in fact demonstrated in these patients after L-NMMA infusion but not after placebo.

In CTTH patients, Ashina et al. [34] also investigated the effect of L-NMMA on increased pericranial muscle hardness and tenderness, which were considered to reflect sensitization of second-order neurons due to prolonged myofascial input. The reduction in the hardness and tenderness of pericranial muscles after L-NMMA infusion in CTTH, demonstrated in this study, supports its potential role as an analgesic in these patients.

The effectiveness of NOS inhibitors on chronic daily 
headache evolving from a previous history of migraine remains to be established. The use of selective inhibitors (for neural and endothelial forms of NOS) may also be particularly helpful in clarifyng the peripheral and central components involved in head pain chronicization in this pathological condition.

\section{Alteration of supraspinal descending facilitating and inhibitory pathways of spinal and trigeminal nociceptive processing}

Monoamine supersensitivity and a condition similar to opiate abstinence have been suggested by Sicuteri et al. [35] to be common features and a different target of the mechanisms in migraine. These abnormalities, according to the authors, can reverse at the end of the attacks. This reiterating may be involved in migraine chronicization.

From a historical point of view, one of the first hypotheses which has been investigated in $\mathrm{CDH}$ is the functional impairment of the endogenous opioid system. More recently, a disturbance of the serotoninergic system was substantiated by several studies in patients with $\mathrm{CDH}$ evolving from a previous history of migraine, particularly in those with chronic drug abuse.

\section{Opioid system and CDH}

A reduction in beta-endorphin levels in patients with continuous migraine and interparoxysmal headache has been observed; these changes were related to the increasing frequency of attacks [36]. However, reduced beta-endorphin levels were not found in the cerebrospinal fluid (CSF) and plasma of patients with CTTH [37]. On the contrary, research carried out by our group some years ago showed lower levels of beta-endorphins in peripheral blood lymphomonocytes of CTTH patients. These cells are considered to be a peripheral window of central neurotransmitter variations, including that of endogenous opioids. The decrease in the levels of these neurotransmitters, related to antinociception, also implied in CTTH a functional impairment of the pathways involved in the processing and control of painful information [38].

Another proof supporting opioid system involvement in $\mathrm{CDH}$ comes from a study from Langemark et al. [39], who showed an increase in the plasma levels of metenkephalin in $\mathrm{CDH}$ patients. This increase was suggested to be related to a compensatory response to the increased serotonin and substance $\mathrm{P}$ release accompanying head pain maintenance in these patients.

\section{Serotoninergic pathways in CDH and their relation to nitric oxide pathway}

A functional impairment of serotoninergic pathways has been proposed in $\mathrm{CDH}$ patients and mainly supported by studies carried out on the platelet model. One of the first studies in this regard demonstrated a reduction in serotonin platelet content in patients affect by CTTH during the cold pressor test compared to control individuals, suggesting a abnormality of 5-HT uptake into platelets and factors which cause release of 5-HT from platelets of the same patients [40]. The reduced serotoninergic function in platelets was also confirmed by the findings of an upregulation of $5-\mathrm{HT}_{2}$ platelet receptors in $\mathrm{CDH}$ patients with a previous history of migraine [41].

A disturbance in intracellular events in platelets of patients with chronic headache associated with drug abuse has also emerged. In research carried out by Hering et al. [42], medication misuse was associated with an alteration in membrane transduction pathways, consisting in decreased responsiveness of the polyphosphoinositide (PPI) system in platelets challenged with thrombin. In the same study, a clear change in the responsiveness of the PPI system emerged upon abrupt withdrawal of abused medication [42]. A more recent study carried out by Srikiatkhachorn and Anthony [43] demonstrated a reduced content and uptake of 5-HT, as well as the upregulation of $5 \mathrm{HT}_{2}$ receptors in platelets of patients with analgesic-induced headache. According to the authors, these findings suggested that analgesic-induced suppression of 5-HT uptake interferes with the function of the pain modulatory system in the brainstem [44].

Collagen induces an increase in L-arginine/NO pathway expression in stimulated platelets, due to the activation of calcium constitutive NOS. Based on these observations, collagen-stimulated platelets can be considered an useful tool for studying NO metabolism in headache patients. Due to the difficulty in assessing NOS activity in vivo in migraine and $\mathrm{CDH}$ patients, the platelet model may help to study NO involvement and the relationship with serotonin metabolism and drug use and misuse in these pathological conditions.

We recently assessed the variations in the activity of the L-arginine/nitric oxide pathway and in platelet cGMP levels in patients affected by $\mathrm{CDH}$ evolving from a previous history of migraine [45]. NO production was measured spectrometrically by the stochiometric transformation of oxyhemoglobin to methemoglobin due to NOS activity Inter-platelet cGMP concentration was assessed by a radioimmunoassay (RIA). NO and cGMP were determnined at the same time with the variations in platelet aggregation response to different collagen concentrations. The intracellular platelet calcium concentration was also determined using fluorescence polarization spectrometry. These parameters were compared 
to those of an age- and sex-matched control group. A reduction in platelet aggregation response for every collagen concentration was found in $\mathrm{CDH}$ patients. The reduction of platelet aggregation was coupled with increased NO and cGMP production. A significant cytosolic $\mathrm{Ca}^{2+}$ concentration increase in patients was found compared to control individuals. This was accompanied by a reduced platelet 5-HT content. We hypothesized that these cGMP-Ca ${ }^{2+}$-mediated events are activated in $\mathrm{CDH}$ patients as a physiologic compensatory mechanism counteracting cytosolic $\mathrm{Ca}^{2+}$ increase, but they are not so efficient to antagonize serotonin depletion by dense bodies in platelets. Whether changes in serotonin and NO metabolism in platelets of $\mathrm{CDH}$ patients reflect similar changes in the CNS is a matter of controversy. However, considering platelets as a peripheral model of monaminergic neurons, a similar alteration of the central serotoninergic pathways in $\mathrm{CDH}$ patients cannot be excluded. The effect of drug withdrawal on NO metabolism in platelets of $\mathrm{CDH}$ patients remains to be established.

Experimental data suggest that a hyposerotoninergic condition can facilitate the NO-induced physiological and pathological responses in cerebral vessels, and therefore, this can be a possible explanation for supersensitivity to NO in migraine patients. This condition can be particularly evident in patients with $\mathrm{CDH}$ evolving from a previous history of migraine [46]. In contrast with the clear evidence for a disturbance of the serotoninergic system in $\mathrm{CDH}$ evolving from a previous history of migraine, recent findings suggest that peripheral 5-HT metabolism and a number of platelet 5HT transporters are on the whole normal in patients with CTTH $[47,48]$.

\section{CDH and drug abuse}

Chronic analgesic use may lower the pain threshold by suppressing the endogenous opioid system, allowing weaker stimuli to evoke headache. Analgesics that chronically suppress the central nociceptive activity upregulate post-synaptic nociceptive receptors [49]. The increased number of nociceptive receptors may lead to an increased perception of pain upon withdrawal of the analgesic or a decrease in its blood level. A similar concept has been invoked to explain the transformation of episodic migraine into $\mathrm{CDH}$.

Increasing activity of the "on cells" in the pain modulation system of the brain stem may enhance the response to any painful and not painful stimuli, resulting in the sensitization of nociceptors in the trigeminal nucleus caudalis. This activity may particularly be enhanced during druginduced headache, a mechanism similar to that occurring during opioid withdrawal. A decrease in platelet serotonin in migraine patients overusing analgesics has been demon- strated; this allows us to hypothesize that analgesic overuse depletes serotonin from storage sites, leading to an increase in the frequency of attacks [40, 44].

As mentioned previously, the 5-HT system was investigated in migraine patients with analgesic abuse by using platelets as neuronal model. A decrease in platelet 5-HT content, which was accompanied by a reduction in platelet dense body number, occurred in these patients compared to controls and patients without analgesic abuse [50]. The presence of large intracytoplasmic vacuoli was also observed. They are formed from the surface-connecting canaliculi system which plays a significant role in platelet secretory response. Dilatation of the canaliculi system implies an excessive release of 5-HT and agrees with our results supporting an hyposerotoninergic state.

The analgesic-induced 5-HT alteration may be a possible mechanism involved in the transformation of headache to chronic form. This concept is supported by the observation that 1-month withdrawal of drugs involved in analgesic-induced headache results in an increase in blood serotonin levels accompanying the reduction in headache frequency [51].

To investigate the effects of chronic analgesic exposure on the central serotonin system and the relation between serotonin system and analgesic consumption, Srikiatkhachorn et al. [52] showed in frontal cortex membranes of male Wistar rats a reduction in the binding sites for $5-\mathrm{HT}_{2} \mathrm{~A}$ and an increase in serotonin transporter due to chronic paracetamol administration. They hypothesized that plasticity of the neurotransmitter system after chronic exposure may lead to the loss of analgesic efficacy, and may produce analgesic-related painful conditions such as analgesic abuse headache.

The neurochemical basis of ergotamine and triptan overuse has not yet been established. As mentioned previously, ergotamine withdrawal reduces summation of second pain in chronic daily headache patients. Moreover, 5-HT supersensitivity and the possible intervention of a "craving for serotonin" was hypothesized to explain the worsening and chronicization of migraine associated with triptan misuse [53].

\section{Conclusions}

Despite the difficult nosography and variability in the clinical features of $\mathrm{CDH}$, one of the most important concerns is the pathophysiological substrate underlying chronicization of pain. Central sensitization has been advocated to explain $\mathrm{CDH}$, both that evolving from a history of migraine and the episodic tension-type headache. 
In the latter, on the basis of the increased tenderness of pericranial muscles and reduced pressor pain threshold in CTTH, Bendtsen [54] affirmed that the qualitatively altered nociception may be attributed in CTTH patients to central sensitization at the level of spinal dorsal horn/trigeminal nucleus due to prolonged nociceptive input from pericranial myofascial tissues. The increased nociceptive input to supraspinal structures may in turn result in supraspinal sensitization. The central neuroplastic changes may affect the regulation of peripheral mechanisms and thereby lead to increased pericranial muscle activity or release of neurotransmitter in myofascial tissue. By such mechanisms, the central sensitization may be maintained even after the initial eliciting factors have been normalized, resulting in the conversion of episodic headache into CTTH. Future research should be aimed at identifying the source of peripheral nociception in order to prevent or reduce just established central sensitization. In the light of this hypothesis, results on efficacy of amitriptyline in reducing headache and myofacial tenderness in CTTH patients should be considered. In these patients amitriptyline seems to elicit its analgesic effect by reducing the transmission of pain stimuli from myofascial tissues, exerting therefore a peripheral antinociceptive action, as well as inducing a segmental reduction of central sensitization [55].

Central sensitization could be also advocated to explain transformed migraine due to maintained peripheral sensitization of vascular structures responsible for maintained trigeminovascular system activation. Alteration of supraspinal pathways involved in the modulation of nociceptive input was demonstrated in these patients.

A functional impairment of the serotoninergic pathway was demonstrated in the platelet model. This impairment consists in the up-regulation of $5 \mathrm{HT}_{2}$ receptors in $\mathrm{CDH}$ patients with a previous history of migraine, especially in those patients where analgesic abuse decreased serotonin content and dilatation of the canaliculi system. This condition causes an excessive release of 5-HT and therefore results in a hyposerotoninergic state.

Recently, our group found increased nitric oxide syn- thase (NOS) activity in patients with $\mathrm{CDH}$, as highlighted by the values of NO and cGMP production, the increased basal and collagen-stimulated cytosolic calcium concentration, and the decreased secretion and reduced concentration of serotonin. These alterations were particularly evident in patients misusing analgesics and ergotamine [45].

Further mechanisms other than the impairment of antinociceptive modulatory pain pathways have been advocated, but only partially investigated in $\mathrm{CDH}$. They involved NMDA receptor activation and NO production and supersensitivity (as shown when using the model of nitroglycerin-induced headache in CTTH), increased and maintained production of SP and CGRP (particularly in transformed migraine) and more recently neurotrophins, in particular nerve growth factor (NGF) [56].

NGF has been demonstrated in experimental models to be involved in acute hyperalgesia and maintaining chronic pain. NGF also provides a prolonged effect potentially through the redistribution of the sodium channels and through the production of PN1 sodium channels in the small neurons of dorsal root ganglia [57]. The potentiating effect of NGF on nociceptive sensory input and NMDA evoked responses suggests its involvement in central sensitization [58]. All this experimental evidence suggests a putative role of NGF and other growth factors in chronic pain. In the future, its involvement should be investigated in $\mathrm{CDH}$ in humans.

The most relevant suggestions on the pathogenetic mechanism of chronic head pain come from in vivo imaging studies. Recent advanced MRI techniques showed signal intensity abnormalities, supporting the occurrence of a biochemical disturbance in brain structures involved in the processing of head pain [59]. Furthermore, positron emission tomography studies may be useful to investigate the potential reiterative activation of some central structures in the brain which have been proposed to be "pain generators" in migraine and which may be more evident in $\mathrm{CDH}$.

Acknowledgements The authors thank Mrs. Nella Perugini (University of Perugia) for technical assistance.

\section{References}

1. Urban MO, Gebhart GF (1999) Central mechanisms in pain. Med Clin North Am 83(3):585-596

2. Coderre TJ, Katz J, Vaccarino AL et al (1993) Contribution of central neuroplasticity to pathological pain: Review of clinical and experimental evidence. Pain 52:259-285

3. McMahon SB, Lewin GR, Wall PD (1993) Central hyperexcitability triggered by noxious inputs. Curr Opin Neurobiol 3:602-610
4. Pertovaara A (1998) A neuronal correlate of secondary hyperalgesia in the rat spinal dorsal horn is submodality selective and facilitated by supraspinal influence. Exp Neurol 149:193-202

5. Watkins LR, Wiertelak EP, Goehler LE et al (1994) Neurocircuitry of illnessinduced hyperalgesia. Brain Res 639:283-299

6. Mendell IM (1996) Physiologic properties of unmyelinated fibre projection to spinal cord. Exp Neurol 16:316-332
7. Post RM, Silberstein SD (1994) Shared mechanisms in affective illness, epilepsy and migraine. Neurology 44[10 Suppl 7]:837-847

8. Fields HL, Heinricher MM, Mason P (1991) Neurotransmitters in nociceptive modulatory circuits. Annu Rev Neurosci 7:309-338

9. Dray A, Urban L, Dickenson A (1994) Pharmacology of chronic pain. Trends Pharmacol Sci 15:190-197 
10. Mao J, Price DD, Hayes RL, Lu J, Mayer DJ, Frenk H (1992) Differential roles of NMDA and non-NMDA receptor activation in induction and maintenance of thermal hyperalgesia in rats with painful peripheral neuropathy. Brain Res 598:271-278

11. Urban MO, Gebhart GF (1998) The glutamate synapse: a target in the pharmacologic management of hyperalgesic pain states. Prog Brain Res 116:382-395

12. Aanonsen LM, Lei S, Wilcox GL (1990) Excitatory aminoacid receptors and nociceptive neurotransmission in the rat spinal cord. Pain 41:309-321

13. Davar G, Hama A, Deykin A, Vos B, Maciewicz R (1991) MK-801 blocks the development of thermal hyperalgesia in a rat model of experimental painful neuropathy. Brain Res 533:327-330

14. Harris JA, Corsi M, Quartaroli M, Arban R, Bentivoglio M (1996) Upregulation of spinal glutamate receptors in chronic pain. Neuroscience 74:7-12

15. Liu H, Mantyh PW, Basbaum AI (1997) NMDA-receptor regulation of substance $\mathrm{P}$ release from primary afferent nociceptors. Nature 386:721-724

16. Felsby S, Nielsen J, Arendt-Nielsen L, et al (1996) NMDA receptor blockade in chronic neuropathic pain. A comparison of ketamine and magnesium chloride. Pain 64:283-291

17. Kristensen JD, Svensson BA, Gordh T (1992) The NMDA-receptor antagonist CPP abolishes neurogenic "wind-up pain" after intrathecal administration in humans. Pain 51:249-253

18. Zahn PK, Umali EF, Brennan TJ (1998) Non NMDA receptor antagonists inhibit pain behaviors in a rat model of postoperative pain. Pain 74:213-224

19. Sang C, Hostetter MP, Gracely RH et al (1998) AMPA kainate antagonist LY293558 reduces capsaicin-evoked hyperalgesia but not pain in normal skin in humans. Anesthesiology 89:1060-1067

20. Markenson JA (1996) Mechanisms of chronic pain. Am J Med 101:S6-S18

21. Mungliani R, Hunt SP (1995) Molecular biology of pain. Br J Anaesthesia 75:1886-1892
22. Hunt SP, Pini A, Evan G (1987) Induction of c-fos like protein in spinal cord neurons following sensory stimulation. Nature 328:1686-1704

23. Meller ST, Gebhart GF (1993) Nitric oxide (NO) and nociceptive processing in the spinal cord. Pain 52:127-136

24. Le Bars D, Villanueva L, Bouhassira D et al (1992) Diffuse noxious inhibitory controls (DNIC) in animals and man. Pathol Physiol Exp Ther 4:55-65

25. Maier SF, Wiertelak EP, Watkins LR (1992) Endogenous pain facilitatory systems - antianalgesia and hyperalgesia. Am Pain Soc J 1:191-198

26. Mansikka H, Pertovaara A (1997) Supraspinal influence of hindlimb withdrawal thresholds and mustard oilinduce secondary allodynia in rats. Brain Res Bull 42:359-365

27. Fusco BM, Colantoni O, Giacovazzo M (1997) Alteration of central excitation circuits in chronic headache and analgesic abuse. Headache 37:486-491

28. Fusco BM (2000) Alteration of the central excitatory circuits in transformed migraine: clinical experimental models. Cephalalgia 20:293

29. Nicolodi M, Sicuteri F (1998) Negative modulators of excitatory amino acids in episodic and chronic migraine: preventing and reversing chronic migraine. Int J Clin Pharmacol Res 18(2):93-100

30. Barabauskas G, Nistri A (1998) Sensitization of pain pathways in the spinal cord: cellular mechanisms. Prog Neurobiol 54(3)349-365

31. Christiansen I, Dangaard D, Lykke, Thomsen L, Olesen J (2000) Glyceryl trinitrate induce headache in migraineurs - relation to attack frequency. Eur J Neurol 7(4):405-411

32. Ashina M, Bendtsen L, Jensen R, Olesen J (2000) Nitric oxide induced headache in patients with chronic daily tension-type headache. Brain 123(Pt 9):1830-1837

33. Ashina M, Lassen LH, Bendtsen L, Jensen R, Olesen J (1999) Effect of inhibition of nitric oxide synthase on chronic tension-type headache: a randomised crossover trial. Lancet 353 (9149):287-289

34. Ashina M, Bendtsen L, Jensen R, Lassen LH, Sakai F, Olesen J (1999) Possible mechanisms of nitric oxide inhibitors in chronic tension-type headache. Brain 122(Pt 9):1629-1635
35. Sicuteri F, Nicolodi M, Del Bianco PL, Del Bene E (1997) Decentralization monoamine supersensitivity of migraine and opiate abstinence: common features and different target mechanisms? Int J Clin Pharmacol Res 17(2-3):67-73

36. Gennazzani AR, Nappi G, Facchinetti F, Micieli G, Petraglia F, Bono G, Monittola C, Savoldi F (1984) Progressive impairment of CSF $\beta$-EP levels in migraine sufferers. Pain 18:127-133

37. Bach FW, Langemark M, Secher NH, Olesen J (1992) Plasma and cerebrospinal fluid beta-endorphin in chronic tension type headache. Pain 51(2):163-168

38. Sarchielli P, Gallai V, Codini M, Franceschini M, Carboni F, Floridi A (1994) Cefalea tensiva: marker biologici In: Puca FM, Scapiccchio P, (eds) Cefalee tensive: classificazione IHS versus DSM-III-R. Possibili fattori psicologici causali. Comitato SISC: Cefalee e Psichiatria. Atti del Convegno di Bari, 21 giugno 1994, pp 131-150

39. Langemark M, Bach FW, Ekman R, Olesen J (1995) Increased cerebrospinal fluid met-enkephalin immunoreactivity in patients with chronic tension-type headache. Pain 63(1):103-107

40. Shimamura T, Takahashi K (1990) Alteration of platelet serotonin in patients with chronic tension-type headache during cold pressor test. Headache 30(9):581-589

41. Srikiatkhachorn A, Govitrapong P, Limthavom C (1994) Up-regulation of 5-HT 2 serotonin receptor: a possible mechanism of transformed migraine. Headache 34(1):8-11

42. Hering R, Gardiner I, Catarci T, Whitmarsh T, Steiner T, De Belleroche J (1993) Cellular adaptation in migraineurs with chronic daily headache. Cephalalgia 13(4):261-266

43. Srikiatkhachorn A, Anthony M (1996) Serotonin receptor adaptation in patients with analgesic-induced headache. Cephalalgia 16(6):419-422

44. Srikiatkhachorn A, Anthony M (1996) Platelet serotonin in patients with analgesic-induced headache. Cephalalgia 16(6):423-426 
45. Sarchielli P, Alberti A, Russo S, Codini M, Panico R, Floridi A, Gallai V (1999) Nitric oxide pathway, $\mathrm{Ca}^{2+}$, and serotonin content in platelets from patients suffering from chronic daily headache. Cephalalgia 19(9):810-816

46. Srikiatkhachorn A, Anuntasethakul T, Maneesri S, Phansuwan-Pujito P, Patumraj S, Kasantikul V (2000) Hyposerotoninergic-induced nitric oxide supersensitivity in the cerebral circulation. Headache 40(4):267-275

47. Bendtsen L, Jensen R, Hindberg I, Gammeltolf S, Olesen J (1997) Serotonin metabolism in chronic tension-type headache. Cephalalgia 17(8):843-848

48. Bendtsen L, Mellerup ET (1998) The platelet serotonin transporter in primary headaches. Eur J Neurol 5(3):277-282
49. Kudrow L (1998) Possible mechanisms and treatment of analgesic-induced chronic headache. In: Diener HC, Wilkinson M (eds) Drug induced headache. Springer, Berlin Heidelberg New York, pp 157-161

50. Srikiotkhachorn A, Manusri S, Govitrapang P, Kasantikul V (1998) Derangement of serotonin system in migrainous patients with analgesic abuse headache: clues from platelets. Headache 38(1):43-49

51. Herig R, Glover V, Pattichis K, Catarci T, Steiner TJ (1993) 5HT in migraine patients with medication induced headache. Cephalalgia 13:410-412

52. Srikiatkhachorn A, Tarasub N, Govitrapong P (2000) Effect of chronic analgesic exposure on the central serotonin system: a possible mechanism of analgesic abuse headache. Headache 40(5):343-350
53. Nicolodi M, Del Bianco PL, Sicuteri F (1997) The way to serotoninergic use and abuse in migraine. Int $\mathrm{J}$ Clin Pharmacol Res 17(2-3):79-84

54. Bendtsen L (2000) Central sensitization in tension type headache - possible pathophysiological mechanisms. Cephalalgia 20(5):486-508

55. Bendtsen L, Jensen R (2000) Amitriptyline reduces myofascial tenderness in patients with chronic tension-type headache. Cephalalgia 20(6):603-610

56. Lewin GR (1993) Nerve growth factor and nocicepetion. Trends Neurol Sci 16:353-359

57. Shu X-Q, Mendell LM (1999) Neurotrophins and hyperalgesia. Proc Natl Acad Sci USA 96:7693-7696

58. Lindsay RM, Harmer AJ (1989) Nerve growth factor regulates expression of neuropeptide genes in adult sensory neurons. Nature 337:362-364

59. Nagesh V, Welch M, Aurora SK, Gelman N, Shanthi G (2000) Is there a brainstem generator of chronic daily headache? J Headache Pain 1(2):67-71 